\title{
Experiments on LHC Long-Range Beam-Beam Compensation and Crossing Schemes at the CERN SPS in 2004
}

\author{
F. Zimmermann, J.-P. Koutchouk, F. Roncarolo, J. Wenninger, CERN, Geneva, Switzerland; \\ T. Sen, V. Shiltsev, FNAL, Batavia, USA; Y. Papaphilippou, ESRF, Grenoble, France
}

\section{Abstract}

Experiments with two prototype long-range beam-beam compensators (current-carrying wires) during the 2004 CERN SPS run explored the efficiency of a proposed longrange beam-beam compensation for the LHC. In addition, the SPS compensators were also used to "simulate" the effect of different planes of crossing at two LHC interaction points. We present the experimental results and compare them with computer simulations.

\section{INTRODUCTION}

Bunches circulating in the Large Hadron Collider (LHC), presently under construction at CERN, will experience up to 120 long-range collisions per turn, where the counterpropagating beams are separated by several times the rms beam size. These long-range collisions give rise to a "diffusive" or dynamic aperture [1] and to an additional tune spread [2]. In order to reduce the latter, the LHC baseline design foresees an alternating crossing scheme, in which the two proton beams are crossed horizontally at two of the four "head-on" interaction points (IPs) and vertically in the remaining two $[3,4]$. This alternating crossing cancels the long-range beam-beam tune shift between pairs of IPs, and it, thereby, assures that bunches at the ends of a bunch train, which do not experience the full number of long-range collisions ("PACMAN" bunches), have nearly the same tunes as nominal bunches [2]. Equal-plane crossing schemes would have other merits, such as zero vertical dispersion, easier long-range compensation, or equal background conditions in the experiments.

A local compensation of the long-range beam-beam collisions in the LHC by means of a current-carrying wire was proposed in $[5,6]$. The transverse distance between wire and beam in units of the rms beam size should be equal to the long-range distance between the two beams. The product of wire current and length needed at one side of one LHC IP is about $80 \mathrm{Am}$ for nominal bunches, increasing to $120 \mathrm{Am}$ for the ultimate LHC intensity.

To study the feasibility of a wire-based beam-beam compensation, an experimental programme with compensator prototypes is underway in the CERN SPS since 2002, when a single device (equipment name "BBLR") was first installed. This single BBLR was used, during 2 years, to "simulate" experimentally the effect of LHC long-range collisions and to benchmark the computer simulations in [7]. More recently, two new BBLR devices were built, each equipped with three wires. A cross section is shown in Fig. 1 (see also [8]). In 2004, one of the two new BBLRs was installed, separated from the existing first-generation BBLR by a phase advance of about $2.6^{\circ}$, which equals the expected average phase advance between compensator and long-range collision points in the LHC. The 3-wire BBLR is movable in the vertical direction over a range of $5 \mathrm{~mm}$.

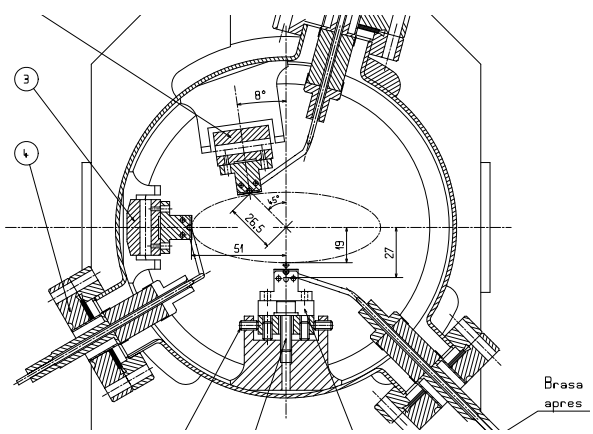

Figure 1: Cross section of the new 3-wire BBLR device installed in the SPS since July 2004 (Courtesy G. Burtin).

For the same normalized beam emittance as in the LHC, $\gamma \epsilon=3.75 \mu \mathrm{m}$, the current of the SPS wire and the distance from the beam in units of rms beam size should be chosen identical. With an effective wire length of $1.2 \mathrm{~m}$, the wire current $I_{\text {wire }}=267$ A then represents the combined effect of all long-range collisions at the two high-luminosity interaction regions of the LHC, and the nominal beam-wire distance is $d_{y}=21.42 \mathrm{~mm}$. The 2004 SPS experiments were performed at $26 \mathrm{GeV} / \mathrm{c}$, where the SPS physical aperture is only about $4 \sigma$ for $\gamma \epsilon=3.75 \mu \mathrm{m}$. To improve the beam lifetime without wire excitation and to more clearly reveal the effect of the wire(s), the emittance can be reduced by scraping. The wire current, $I_{\text {wire }}$, and beam-wire distance, $d_{y}$, are then scaled linearly with the emittance and with the square root of the emittance, respectively.

Below we present the highlights of the 2004 SPS experiments. Some details can be found in [9].

\section{COMPENSATION}

Powering a single BBLR models the effect of the uncompensated LHC long-range collisions. The observed closedorbit orbit distortion and tune shifts induced by the wire, with typical magnitudes of a few 100s of $\mu \mathrm{m}$ and several $10^{-3}$, respectively, agree with analytical calculations and simulations [8]. The nonlinear effects of the wire manifest themselves in beam losses, in a reduction of the dynamic aperture, as evidenced by a shrinking emittance and by a degradation of the beam lifetime, in a decreased decoherence time and in a change of the detuning with amplitude $[8,10]$. Several scans were performed of the wire current and of the beam-wire distance, corresponding to LHC beam current and crossing angle, respectively $[8,10]$. Figure 2 shows a 2004 single-wire measurement, where the beam lifetime was recorded as a function of the beam-wire 
separation. The dependence is perfectly fitted by a 5 th order power law of the form $\tau \approx 5 \mathrm{~ms}(d / \sigma)^{5}$. A naive extrapolation to the typical LHC separation of $9.5 \sigma$ would yield a beam lifetime of about 6 minutes. In a similar experiment at the Tevatron, where the electron lens (TEL) was used to mimic a "wire", the beam lifetime increased as the third power of the beam-TEL distance [11]. The different power law may be related to the working point in tune diagram; the low powers remain as yet unexplained.

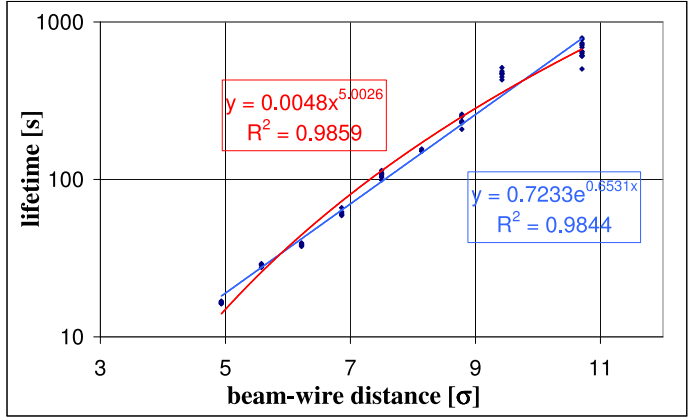

Figure 2: Fitted SPS beam lifetime as a function of the beam-wire distance, with a single-wire excitation of $190 \mathrm{~A}$, tunes $Q_{x}=0.3208, Q_{y}=0.2914 ; \gamma \epsilon_{x} \approx \gamma \epsilon_{y} \approx 1.4 \mu \mathrm{m}$, $\sigma \approx 1.56 \mathrm{~mm}$. Beam and wire were separated vertically.

In 2004, two SPS experiments on wire compensation were conducted. The typical total beam intensity was $3.5 \times 10^{11}$ protons distributed in 12 bunches. All experiments were performed on the parasitic SPS cycle with beam present for a period of only 2 to $4 \mathrm{~s}$. On 29/07 and 30/07/2004 the effect of the first BBLR was compensated by the new BBLR (BBLR2). For this study, the normalized transverse beam emittances were 4-6 $\mu \mathrm{m}$. Tune scans were conducted around the standard SPS working point for LHC beam $\left(Q_{x, y} \approx 0.17\right)$ and around the tunes foreseen for the LHC itself $\left(Q_{x, y} \approx 0.31\right)$. Figure 3 (right picture) shows the measured beam-current lifetime of the bare machine (no BBLR excitation), with only one BBLR excited, and with the compensating BBLR also turned on, as a function of vertical tune near the LHC working point. The left picture illustrates the trace followed in the tune diagram. The single BBLR reduced the beam lifetime from about $250 \mathrm{~s}$ to $50 \mathrm{~s}$. The compensation restored the original lifetime, except for tunes below 0.285 and 0.275 , i.e., the regions of the 7 th and 4 th order resonances. The imperfect compensation for low tunes could also be an artifact of drifting machine conditions. In all cases, the beam lifetime dropped as the tune approached the 3rd integer resonances, at $0.32-0.33$. This lifetime reduction must be driven by the natural nonlinearities of the SPS, since it is only weakly affected by the BBLR. The vertical mobility of the new BBLR allowed measuring the sensitivity of the compensation to transverse wire position. Figure 4 shows the inverse lifetime inferred from the losses measured over $2.4 \mathrm{~s}$ as a function of the offset between the two wires. Also shown are the simulated loss rates at each offset in arbitrary units as calculated with the code BBSIM developed at FNAL [12]. For comparison the simulated and measured loss rates with only the
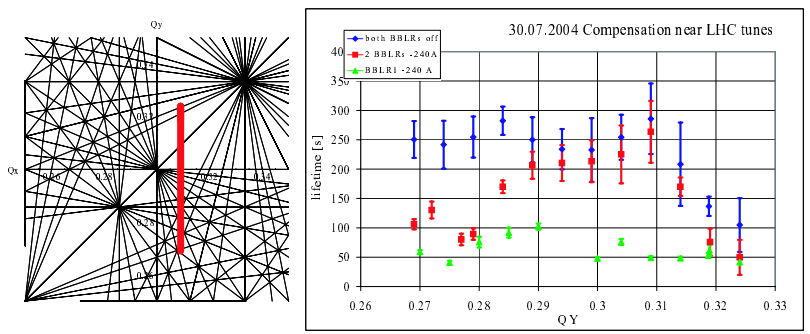

Figure 3: Left: tune path traced during the SPS wirecompensation experiment on 30/07/04., and resonance lines through 11th order; right: measured beam lifetime vs. vertical tune without BBLR (blue diamonds), with one BBLR at 240 A (green triangles) and with both BBLRs excited so that they compensate each other (red squares).

first wire BBLR1 activated are given by the two superimposed straight lines. The measurements showed that there was no compensation with offsets beyond $2.5 \mathrm{~mm}$ since the losses with both wires exceeded the loss due to BBLR1 alone. The simulations predicted the compensation to be inefficient with offsets above $3 \mathrm{~mm}$, in reasonable agreement with the data.

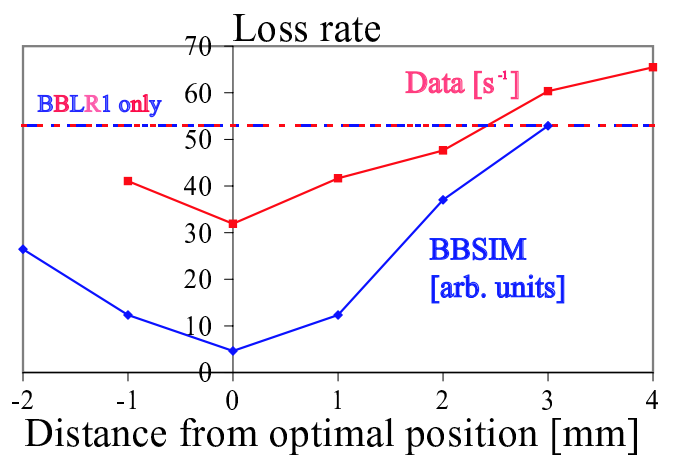

Figure 4: Relative SPS loss rate in $\mathrm{s}^{-1}$, as measured over $2.4 \mathrm{~s}$, and simulated loss rate in arbitrary units vs. the vertical position of the compensating second wire. Measured and simulated loss rates for a single wire are indicated by the two superimposed straight lines.

A second compensation experiment was performed on $02 / 09 / 2004$, with a smaller emittance of about $1.5 \mu \mathrm{m}$. The natural beam lifetime $\tau$ was a factor 20 longer than for the previous experiment with larger emittances, and it dropped by a factor 2 when a single SPS wire was excited. The compensation by the second wire not only restored, but even improved the lifetime by about $10 \%$ compared to that of the bare machine. The strong dependence of the beam lifetime on the emittance is reminiscent of the dependence $\tau \propto 1 / \epsilon^{2}$ observed for antiprotons at the Tevatron [13].

\section{CROSSING SCHEME}

On 26/08/2004, an experiment on the crossing planes was conducted for the nominal emittance. Three configurations were implemented. Due to constraints by the physical aperture and the different distance of the horizontal and vertical wire from the center of the chamber (about $55 \mathrm{~mm}$ 
and $20 \mathrm{~mm}$, respectively), a pure alternating crossing could not be realized. Instead a mixed scheme was chosen, modeling horizontal crossing at one wire and 45-degree crossing at the other, by exciting both wires at the same current. Equal-plane crossings were modeled by exciting only one of the two wires at twice its original strength. For completeness, and to observe a larger effect on the beam lifetime, the first configuration was also tested at twice the strength, which simulates a two times higher beam intensity. For all wire configurations, we measured the beam lifetime as a function of the vertical tune, which was varied between 0.26 and 0.33 . Over most of the scanned tune range, the horizontal-horizontal crossing (BBLR2 excited at -240 A) exhibited the best beam lifetime, the pure 45degree crossing (BBLR1 at $240 \mathrm{~A}$ ) the second best, and the mixed crossing (BBLR1 at 120 A, BBLR2 at -120 A) the lowest. At the two ends of the scan range, near the 7th and 3rd integer resonance, respectively, the pure 45-degree crossing scheme was most robust, while for all others the lifetime strongly decreased here, possibly due to lattice nonlinearities. The lifetime without any wire excitation was comparable to that of the mixed-crossing case.

On 09/11/04, a second experiment was performed with reduced beam-wire distance and smaller emittance. One of the wires (BBLR2) had been rotated prior to this experiment, in order to allow for shorter transverse distances. The three configurations of Fig. 5 could then be realized. Again, it was not possible to implement a pure horizontal-vertical crossing. Instead a $45^{\circ}-135^{\circ}$ inclined hybrid crossing [14] was modeled and its performance could be compared with that of a vertical-vertical or $45^{\circ}-45^{\circ}$ crossing.

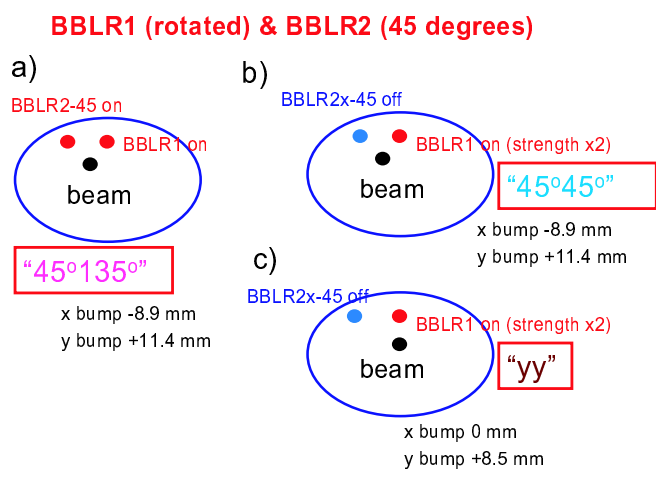

Figure 5: Approximations of different crossing schemes on 09/11/2004. Configuration a) models $45^{\circ}-135^{\circ}$ inclined hybrid collision [14], b) a double $45^{\circ}$ hybrid crossing and c) a pure vertical-vertical crossing.

Figure 6 displays the simulated dynamic aperture for these three configurations. The pure $45^{\circ}-45^{\circ}$ crossing has the smallest dynamic aperture. At vertical tunes of 0.29 or lower the vertical-vertical crossing is best, while at higher tunes the inclined-hybrid scheme yields the largest dynamic aperture. For completeness, the simulation results for a pure horizontal-vertical crossing are also indicated.

The measured beam lifetimes as a function of vertical tune are presented in Fig. 7. The lifetime was lowest for

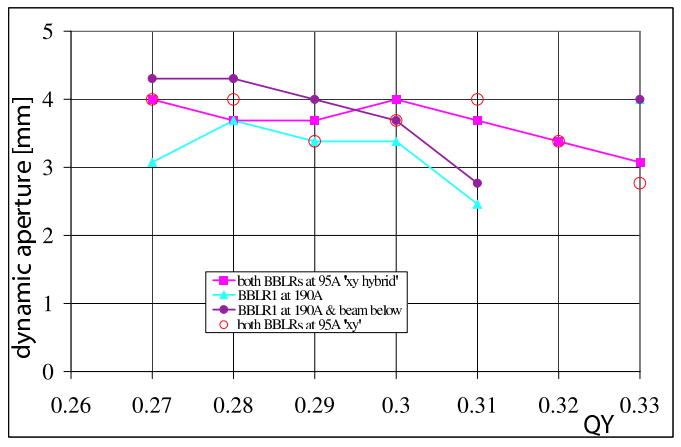

Figure 6: Dynamic aperture simulated by the WSDIFF code (at $\beta \approx 50 \mathrm{~m}$ ) as a function of vertical tune keeping $Q_{x}=0.32$, for the SPS wire configurations of Fig. 5 .

the $45^{\circ}-45^{\circ}$ crossing, the inclined hybrid crossing best for tunes above 0.3 and the pure vertical-vertical crossing for lower tunes, - all consistent with the simulations in Fig. 6.

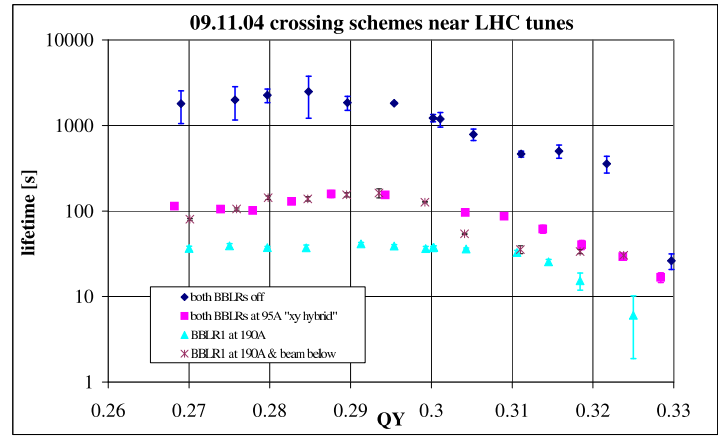

Figure 7: Beam lifetime measured as a function of the vertical tune for the three SPS wire configurations of Fig. 5. The horizontal tune was held constant at $Q_{x} \approx 0.32$.

\section{CONCLUSIONS}

The LHC beam lifetime may be degraded by long-range collisions. The efficiency of the proposed compensation was demonstrated. The beam lifetime was shown to vary with crossing scheme. Experiments and simulations are mostly compatible, but a few results are still unexplained.

\section{REFERENCES}

[1] J. Irwin, SSC-233 (1989).

[2] D. Neuffer, S. Peggs, SSC-63 (1986).

[3] W. Herr, CERN/SL/90-06-AP (1990).

[4] O. Bruning et al. (eds.), CERN-2004-003 (2004).

[5] J.-P. Koutchouk, LHC Project Note 223 (2000).

[6] J.-P. Koutchouk, PAC 2001 Chicago (2001).

[7] Y. Papaphilippou et al., PRST-AB 2:104001 (1999).

[8] J.-P. Koutchouk et al., EPAC'04 Lucerne (2004).

[9] F. Zimmermann, "Beam-Beam Compensation Schemes," HHH-2004 Workshop, CERN (2005).

[10] F. Zimmermann, Factories '03 Workshop, Stanford (2003).

[11] F. Zimmermann et al., CERN AB-Note-2004-041 (2004).

[12] T. Sen, "Beam-Beam Simulations for Hadron Colliders," HHH-2004 Workshop, CERN (2005).

[13] V. Shiltsev et al., "Beam-Beam Effects in Tevatron Run II," PAC'05 Knoxville (2005).

[14] K. Takayama et al., Phys. Rev. Let. 88, 14, 144801-1 (2002). 